\title{
ABORTIVE FLOWER-CLUSTER MUTANT IN TOMATOES
}

An unfruitful tomato plant of the variety Kokomo was found in a commercial field. It was of indeterminate type and the plant reached approximately 6 feet in length. At the position where a normal flower cluster should have been, only an aborted cluster a few millimeters in length, could be seen (fig. 1).

As in a normal plant, the abortive cluster occurred regularly every 2 or 3

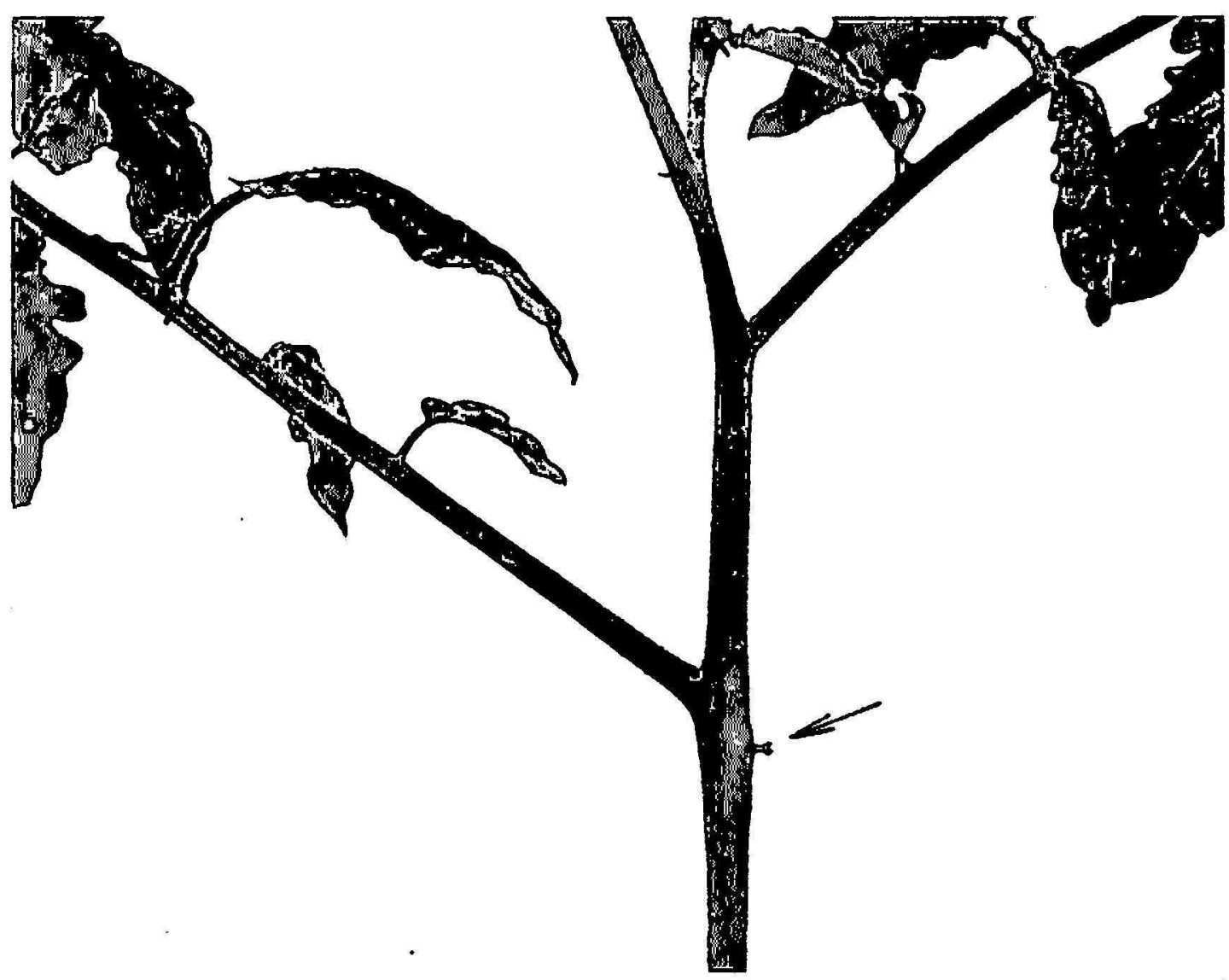

Fici 1.-The abortive flower clusters of the tomato mutant. 
$\circlearrowright$

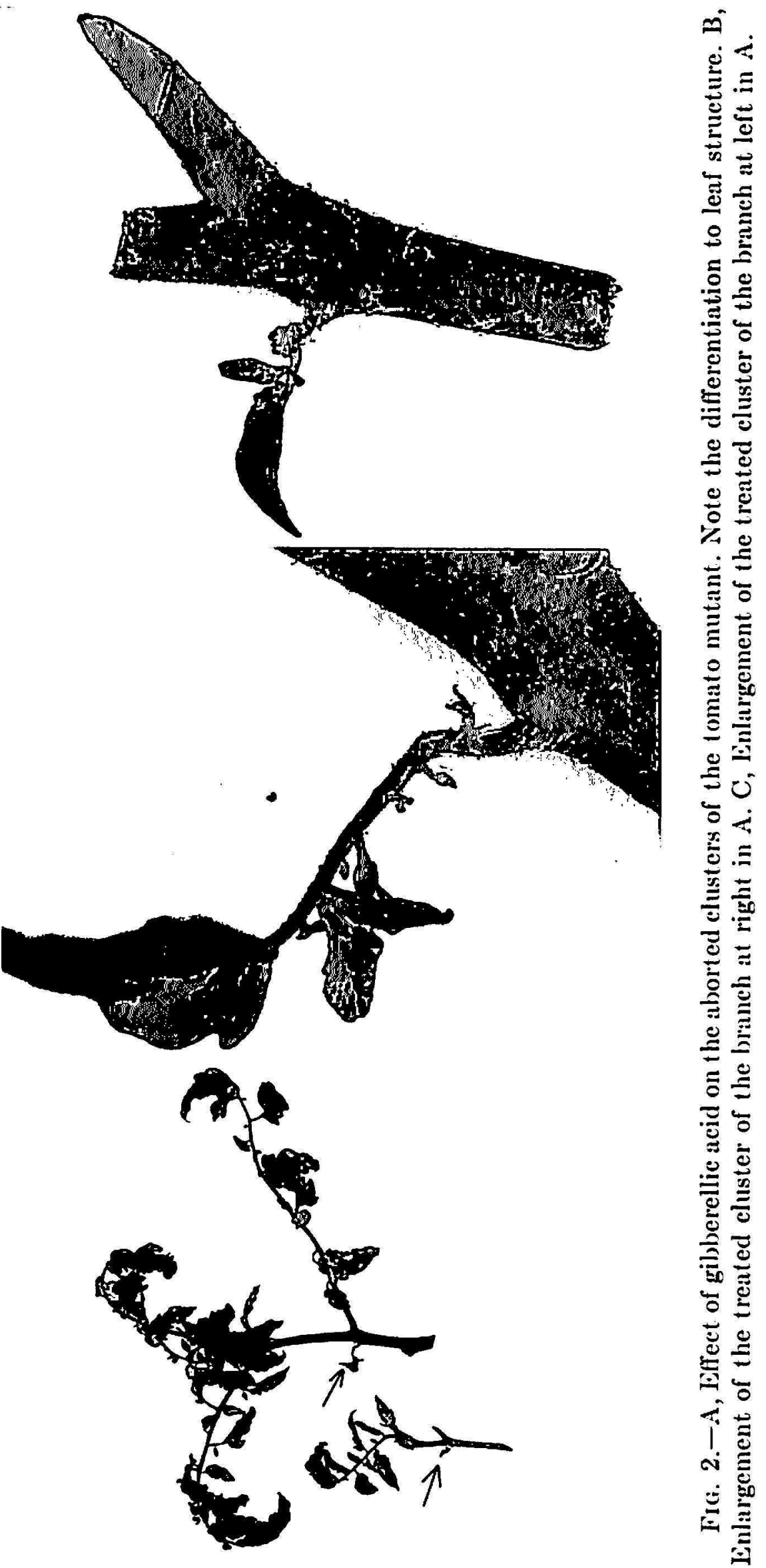


leaves. This mutant represents an extreme case of sterility showing the complete absence of the male and female parts of each flower. Rick and Robinson ${ }^{1}$ found a similar, if not the same mutant, in a culture of unknown pedigree.

Successful approach-grafting with the Manalucie variety, using the mutant either as seion or stock, failed to alter either the normal flower development of the Manalucie plants or the abnormal cluster of the mutant.

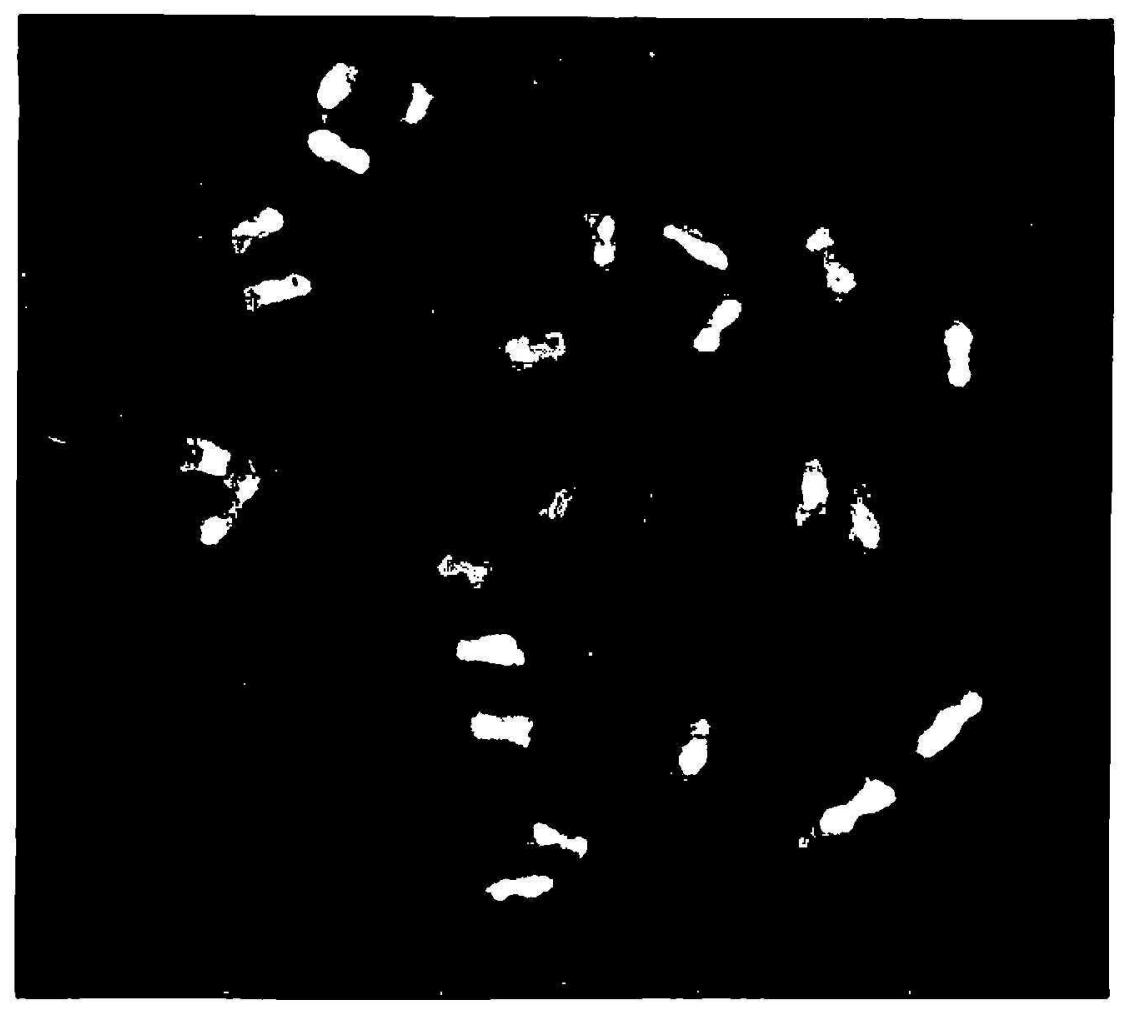

FIG. 3.-Chromosomal feature of the mutant indicating its diploid nature (a negalive photograph).

The aborted flower clusters were treated woekly for is wecks with gibberellic acid in concentrations of 25,50 , and 100 p.p.m. The lowest (oncentration did not show any visible effect. Treatment with the 100 p.p.m. gibberellic arid stimulated the differentiation to leaf strueture and cularged visible aborted individual flowers (fig. 2).

Only one of the individual flowers of the treated clusters developed to a practically full leaf while the other flowers became visible and grew somewhat. Therefore, treatment of the mutant with gibberellic arid failed to produce normal flowers.

Clonal propagations of the mutant for several generations in the green-

เ Rick, C. M., and Robinson, J., Inherited defects of floral st ructure affecting fruit fulness in Lycopersicon esculentum, Amer. J. Bot. 38 (8) 639-52, 1951. 
house and the field did not alter the aborted clusters, indicating the stability of this mutant under the various environmental conditions.

Chromosomal studies showed that the mutant plant was a normal diploid (fig. 3), indicating that the mutant is of genic nature.

The inheritance mechanism of this mutant is difficult to study; however, it is likely to be due to a single gene. 